\title{
Nose Detection and Breathing Monitoring in Thermal Images
}

\author{
Jin-Sup Eom ${ }^{1}$, Mi-Sook Park ${ }^{2}$, Hye-Ryeon Yang ${ }^{3}$, Young-Ji Eum ${ }^{4}$ \\ and Jin-Hun Sohn ${ }^{\text {* }}$ \\ ${ }^{1}$ Department of Psychology, Chungbuk National University, Cheongju, Korea \\ ${ }^{2}$ Department of Rehabilitation Counseling Psychology, Seoul Hanyoung \\ University, Seoul, Korea \\ ${ }^{3}$ Department of Cognitive Science, Pusan National University, Busan, Korea \\ ${ }^{4,5}$ Department of Psychology/Brain Research Institute, Chungnam National \\ University, Daejeon, Korea \\ 1jseom2003@hanmail.net, 2misook_park1121@hanmail.net,

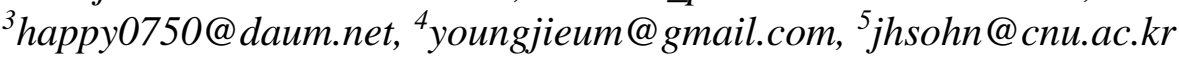

\begin{abstract}
This study proposed a method of nose and nostril detection in thermal images using the Viola-Jones method. Since thermal images show facial features with unclear boundaries, using the Viola-Jones method to identify features results in hits and false alarms. To eliminate false alarms, the face was first detected by setting size and temperature constraints. Next, location constraints were added to precisely locate the nose and nose holes. After detecting the nose and nose holes, template matching was used to track the nose holes, and breathing was monitored by calculating the average temperature in the nostril region. When the proposed method was applied to thermal images captured for one minute each from 20 subjects, the nose and nose holes of all 20 subjects were detected with $100 \%$ accuracy within 3 seconds. The nose holes were also successfully tracked for all cases. Breathing rates were compared for measurements obtained from average nostril temperature and from a monitoring device. This revealed a match for 16 out of the 20 subjects, and only one different measurement for 2 subjects.
\end{abstract}

Keywords: Nose detection, Breathing monitoring, Thermal image, Haar-like feature

\section{Introduction}

Recently, there has been an increase in the use of facial thermal images in human engineering and psychology studies. These studies cover various topics such as facial expression recognition, lie detection, and breathing monitoring.

Many studies have attempted facial expression recognition using facial images. However, facial images can look different depending on lighting, and expressions cannot be detected when there is little or no light. Facial thermal images, on the other hand, produce the same results regardless of lighting angle and intensity. Some studies have used facial thermal images for facial expression recognition[1-4]. As the facial muscles shift to make different expressions, the facial surface temperature changes because it is related to the distribution of muscles and blood vessels, and the positioning of eyes, nose and mouth. Facial expression recognition can be performed with a single thermal image[1], or by utilizing changes in facial surface temperature[2]. To detect facial expression, it is necessary to first locate the nose, eyes, and mouth. Facial feature positions are needed to determine the surface temperature distribution of the face.

Received (October 8, 2017), Review Result (November 28, 2017), Accepted (December 20, 2017)

*Corresponding Author 
In lie detection, facial surface temperature can be used as a dependent value[5-7]. Liars tend to be anxious over being caught, and lie detectors measure physiological reactions arising from such anxiety. Stimuli directly related to the lie produces arousal in liars, and there are some lie detectors that measure physiological reactions associated with arousal. Anxiety affects temperature in areas around the eyes and the forehead[8], and arousal also changes temperature near the eyes[6].

Important factors in breathing monitoring include polygraph, sleep research, sports training, sudden infant death, and monitoring of patients[9]. To monitor breathing, expansion/contraction sensors can be attached to the chest and abdomen, or temperature sensors below the nose. If thermal images are used, breathing can be monitored from a longer distance without the use of sensors[10]. Cold air enters the nose when inhaling, and relatively warmer air flows out through the nose when exhaling. This difference in temperature near the nose holes can be measured through a thermal imaging camera.

Studies on facial expression recognition, lie detection, and breathing monitoring require observation of specific facial areas in real time. In the case of facial expression recognition, temperature changes above the nose (near the eyes) and below (near the mouth) must be monitored. For lie detection and breathing monitoring, temperature changes must be monitored near the eyes and nose holes, respectively. As such, an algorithm is required to identify facial features in thermal images. It is particularly important to locate the tip of the nose and nose holes for more accurate results in facial expression recognition, lie detection, and breathing monitoring. This will facilitate the identification of other facial features.

Compared to visual images, facial features in thermal images have unclear boundaries. Thus, it is inappropriate to apply algorithms for visual images to thermal images[2]. A few studies have begun developing algorithms for nose detection in thermal images[11, 12]. Al-Khalidi and his colleagues used the Prewitt operator to detect the boundaries of the face in the first thermal image, and then located the eyes and nose in the identified face[11]. The inner end of one eye was marked as the eyes are generally the warmest facial points. The inner end of the remaining eye was also marked by finding the second warmest region. The region with the lowest facial temperature was located below the two marked points, and this corresponded to the tip of the nose. Once the tip of the nose was found, a circle was placed around the tip. This area was then subject to tracking. However, there may be some difficulty in accurately locating the nose since this method involves locating the region with the lowest facial temperature below the eyes without searching for the nose directly.

Hanawa and his colleagues directly located the nose in thermal images by using Haarlike features and the Adaboost algorithm developed by Viola and Jones[12-13]. They used thermal images of 9 subjects recorded for 10 seconds each at 2700 frames. Using the nine-fold cross-validation procedure, 2400 frames were used in training, and 300 frames for nose detection. The accuracy rate was $64.4 \%$, but this included frames with multiple detected areas. In other words, false alarms accounted for $85.7 \%$. When frames having multiple detected areas were excluded, the accuracy rate fell below $15 \%$.

Al-Khalidi and his colleagues allowed effective tracking by first identifying the nose while Hanawa and his colleagues used a more direct method in finding the nose. This study combines the advantages of these two studies, and proposes a method that identifies the nose from initial thermal images followed by tracking in subsequent thermal images. As shown in the study by Hanawa, Haar-like features and the Adaboost algorithm result in a high false alarm rate. In this study, the face, nose, and lower part of the nose including nose holes were identified based on Haar-like features and the Adaboost algorithm.

The three identified features were then combined to form the lower part of the nose. Next, template matching was employed to track the lower part of the nose, and breathing 
rates were monitored with the average temperature of this region. To prevent confusion, the lower part of the nose shall be referred to as nose holes in the rest of the paper.

\section{Methods}

This study developed a method to search for nose holes in thermal images, verified the developed method, and explored the possibility of applying the method to breathing monitoring. The three stages involved are as follows. The first stage was training of the face, nose, and nose holes. In the second stage, a detection procedure for nose holes was established based on the training results. The third stage verified the search procedure established in the previous stage, and determined if breathing monitoring was possible. Figure 1 gives a brief outline of the search procedure for the nose and nose holes.

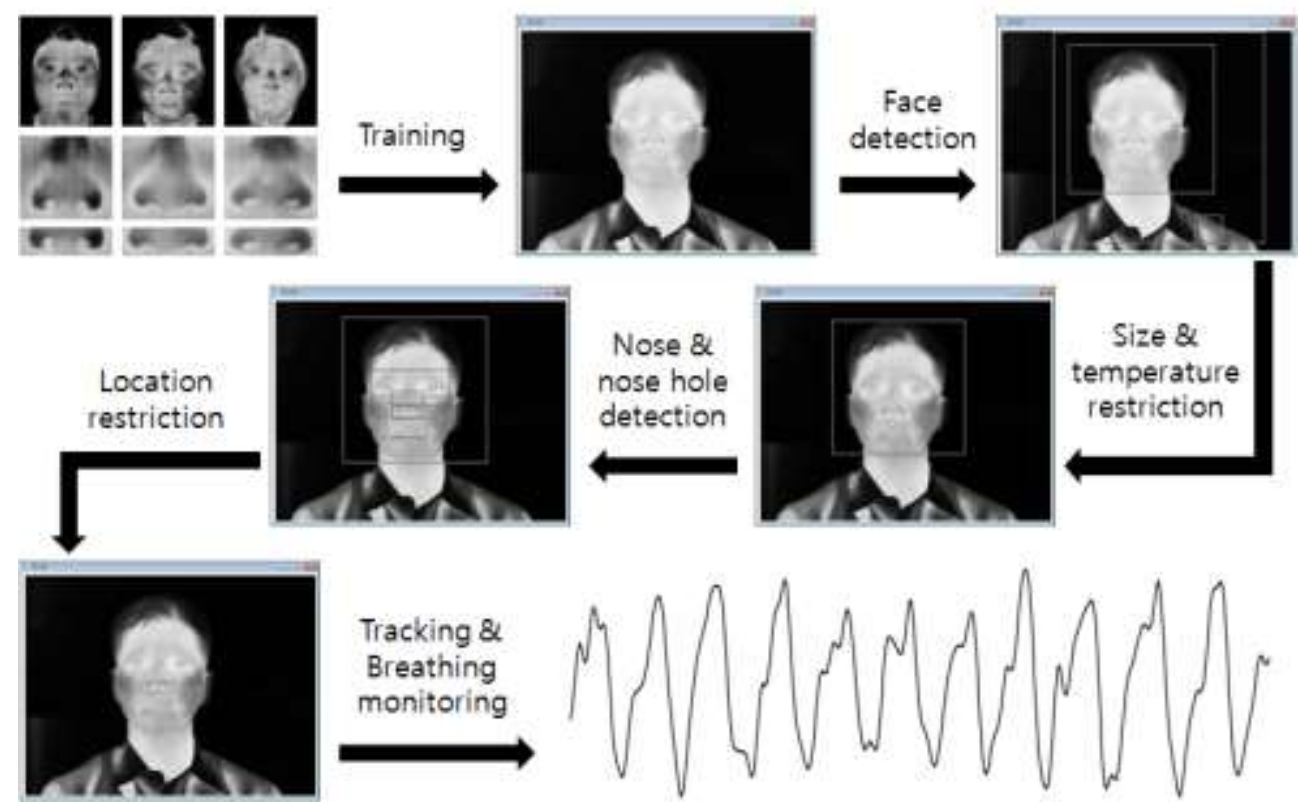

Figure 1. Procedure of Nose Hole Detection and Breathing Monitoring

\subsection{Training of Face, Nose, and Nose Holes}

Thermal images of the upper bodies of 278 subjects were used as training data, and this shall be referred to as the training sample. The subjects were aged 19.9 years old on average (ranging from 13 to 31), and there were 127 men. Thermal images were recorded with a H2640 (NEC-Sanei, Japan), which features a temperature range of $-20 \sim 60^{\circ} \mathrm{C}$, $640 \times 480$ pixel resolution, capture wavelength of $8 \sim 16 \mu \mathrm{m}$, and a thermal resolution of $0.06^{\circ} \mathrm{C}$. Using Equation (1), temperature obtained from thermal images was converted into grayscale. Since the average surface temperature of the face is approximately $33^{\circ}, \mu$ was set as 33 to distinguish facial features more easily.

$f(x)=\frac{255}{1+e^{-(x-\mu)}}$

As can be seen from the converted thermal images of 278 subjects in Figure 1, the face area was selected face training data, the nose areas as nose training data, and the lower 
region of the nose including nose holes as nose hole training data. When performing training for the various facial features, 800 faceless thermal images were used as background images. Facial feature training was conducted with Haartraining, including OpenCV 2.4.5, at $24 \times 24$ for the face, $24 \times 20$ for the nose, and $36 \times 12$ for nose holes.

\subsection{Establishment of Detection Procedure for Nose and Nose Holes}

The detection procedure for nose holes was established using 20 -second thermal images of the upper bodies of 20 subjects (10 males, aged 19 to 46), and this sample shall be referred to as the exploratory sample. While capturing thermal images, the subjects were seated $1.5 \mathrm{~m}$ away in front of the thermal imaging camera and asked to focus on a marked point. Thermal images were captured with $\mathrm{H} 2640$ at 5 frames per second.

Haar-like features and the Adaboost method tend to produce many false positives. This issue was resolved by the following procedure. First, the face is detected in the input thermal image. Second, the nose and nose holes are detected in the face region. Third, the nose and nose hole areas in the center of the face is selected among the multiple detected areas. Fourth, nose and nose hole pairs with nose hole areas below nose areas are selected among nose areas and nose hole areas satisfying the aforementioned conditions.

\subsection{Verification of Detection Procedure and Breathing Monitoring}

To verify the detection procedure developed for the nose and nostrils, thermal images were captured for 20 new subjects (10 males, aged 19 39), and this sample shall be referred to as the validity sample. The subjects were seated $1.5 \mathrm{~m}$ away in front of the thermal imaging camera, and sensor belts were placed around their chests to monitor breathing. They were instructed to focus on a marked point, while breathing rates were recorded for 60 seconds. Thermal images were captured with $\mathrm{H} 2640$ at 5 frames per second. Breathing rates were monitored via TSD101B (Biopac System Inc., USA) sensors and RSP100B (Biopac System Inc., USA) amplifiers.

The nose and nose holes of each subject in thermal images were identified using the proposed method of this study. After detecting the nose and nose holes, the grayscale value of the nose hole region was used in the tracking of nose holes. Nose hole tracking was performed using the cvMatchTemplate() function in OpenCV 2.4.5. The average temperature of the nose hole region was calculated while tracking, and the possibility of breathing monitoring was assessed in consideration of periodic temperature changes.

\section{Results}

\subsection{Detection Procedure for Nose and Nose Holes}

3.1.1. Face Detection: Thermal images in the exploratory sample were converted into grayscale by Eq. 1. The average surface temperature of the face is approximately $33^{\circ}$, with slight differences by individual. When Equation (1) is used in grayscale conversion for the thermal image of a subject with low surface temperature, it becomes difficult to detect the nose and nose holes as the face area will mostly be black. For a person with high surface temperature, most of the face area will be white, making it equally difficult to detect the nose and nose holes. The face was detected by applying Equation (1) to the first thermal image of each subject for grayscale conversion. After calculating the average surface temperature of the face, 
this value was used to replace $33^{\circ}$ in Equation (1) for grayscale conversion of the remaining thermal images.

Face detection was performed using the results of face training and the cvHaarDetectObjects() function included in OpenCV 2.4.5. To evaluate the accuracy of face detection, faces were identified in all thermal images $(20$ subjects $\times$ 20 seconds $\times 5$ frames $=2000$ frames). As a result, 1.3 face areas were detected on average from a single thermal image. As presented in Table 1, the miss rate was $0.0 \%$ for multiple detected face areas, implying that the actual face was included in all occurrences. The correct detection rate for the actual face only was $71.8 \%$, while the false alarm rate was $28.2 \%$.

Table 1. Detection Results of Face, Nose, and Nose Hole for Exploratory Sample

\begin{tabular}{|c|c|c|c|c|c|c|c|c|c|}
\hline \multirow{2}{*}{ Subjects } & \multicolumn{3}{|c|}{ Face } & \multicolumn{3}{|c|}{ Nose } & \multicolumn{3}{|c|}{ Nose hole } \\
\hline & $\mathrm{CD}^{\mathrm{a}}$ & $\mathrm{FA}^{\mathrm{b}}$ & $M^{c}$ & $\mathrm{CD}$ & FA & M & $\mathrm{CD}$ & FA & $\mathrm{M}$ \\
\hline 1 & 82 & 18 & 0 & 36 & 64 & 0 & 0 & 100 & 0 \\
\hline 2 & 100 & 0 & 0 & 32 & 68 & 10 & 0 & 100 & 0 \\
\hline 3 & 100 & 0 & 0 & 12 & 88 & 0 & 34 & 66 & 0 \\
\hline 4 & 100 & 0 & 0 & 24 & 76 & 0 & 0 & 100 & 0 \\
\hline 5 & 100 & 0 & 0 & 38 & 62 & 0 & 40 & 58 & 2 \\
\hline 6 & 100 & 0 & 0 & 28 & 72 & 0 & 30 & 22 & 40 \\
\hline 7 & 98 & 2 & 0 & 16 & 84 & 0 & 24 & 70 & 6 \\
\hline 8 & 24 & 76 & 0 & 66 & 34 & 0 & 4 & 96 & 0 \\
\hline 9 & 100 & 0 & 0 & 54 & 46 & 0 & 28 & 54 & 22 \\
\hline 10 & 100 & 0 & 0 & 30 & 62 & 38 & 0 & 100 & 2 \\
\hline 11 & 70 & 30 & 0 & 6 & 94 & 0 & 0 & 100 & 0 \\
\hline 12 & 88 & 12 & 0 & 12 & 88 & 0 & 8 & 92 & 0 \\
\hline 13 & 60 & 40 & 0 & 2 & 98 & 0 & 0 & 100 & 0 \\
\hline 14 & 48 & 52 & 0 & 48 & 52 & 0 & 16 & 84 & 0 \\
\hline 15 & 2 & 98 & 0 & 0 & 100 & 0 & 0 & 100 & 0 \\
\hline 16 & 0 & 100 & 0 & 0 & 100 & 0 & 4 & 96 & 0 \\
\hline 17 & 100 & 0 & 0 & 0 & 16 & 100 & 0 & 62 & 100 \\
\hline 18 & 0 & 100 & 0 & 0 & 100 & 0 & 6 & 94 & 0 \\
\hline 19 & 64 & 36 & 0 & 6 & 94 & 0 & 54 & 46 & 0 \\
\hline 20 & 100 & 0 & 0 & 2 & 98 & 0 & 8 & 92 & 0 \\
\hline Mean & 71.8 & 28.2 & 0 & 20.6 & 74.8 & 7.4 & 12.8 & 81.6 & 8.6 \\
\hline Std. Dev. & 37.3 & 37.3 & 0 & 20.2 & 24.2 & 23.5 & 16.4 & 23.0 & 23.7 \\
\hline
\end{tabular}

Two constraints were imposed to detect the actual face in multiple detected areas. One constraint was the size of the face. The size of nose holes had to be at least $36 \times 12$ pixels, and the size of the nose including nose holes had to be $36 \times 30$ pixels or larger. An analysis of face proportions in the training sample revealed that the minimum ratio was 0.17 for the nose to face width, and 0.15 for height. For the nose to be at least $36 \times 30$ pixels, the face has to be approximately $211 \times 200$ pixels. A 
minimum face size of $211 \times 211$ pixels was used as a constraint. Another constraint was the proportion of the background included in the face region. Since the facial temperature of the face is $31^{\circ}$ or higher, areas lower than $31^{\circ}$ including hair can be considered as the background. The proportion of pixels with a temperature below $31^{\circ}$ was calculated for the training sample. The average proportion was $51.3 \%$. For multiple detected areas, the area in which pixels falling below $31^{\circ}$ accounted for close to $50 \%$ was selected as the actual face. With these two constraints, the face was accurately identified in all 2000 frames.

3.1.2. Detection of Nose and Nose Holes: Nose and nose holes were detected in the identified faces in order to calculate the accuracy rate of nose and nose hole detection. On average, one thermal image had 2.3 nose areas and 2.7 nose hole areas. As shown in Table 1, nose detection had a miss rate of $7.4 \%$, a correct detection rate of $20.6 \%$, and a false alarm rate of $74.8 \%$. Nose hole detection had a miss rate of $8.6 \%$, a hit rate of $12.8 \%$, and a false alarm rate of $81.6 \%$. These figures were similar to the results obtained by Hanawa et al., [12].

The first thermal image for each subject was used in calculating for grayscale conversion. Face areas were thus identified from subsequent thermal images, followed by detection of the nose and nose holes. Two constraints were imposed to determine which of the multiple selected areas contained the actual nose and nose holes. One was a constraint on the location of the nose and nose holes, which can be found in the center of the face. Among the areas with multiple detection of nose and nose holes, those containing detected areas in the upper $20 \%$ and lower $20 \%$ of the face were excluded. Another constraint was the relative position of the nose and nose holes. Among the identified nose areas and nose hole areas, nose and nose hole pairs with nose hole areas below nose areas were selected. The pair with a lower temperature was selected if there were multiple pairs meeting the above constraints. If the nose and nose holes were undetected in the second thermal image of subjects, the procedure was repeated for subsequent thermal images.

With these constraints, the nose and nose holes were accurately detected for 19 out of the 20 subjects. The features were accurately detected in the second thermal image for 14 out of the 19 subjects, and the most number of images required to complete the detection procedure was five. The nose and nose holes were undetected for one of the subjects, as shown in Figure 2, because similar temperatures were observed for the nose and surrounding areas.

(A)

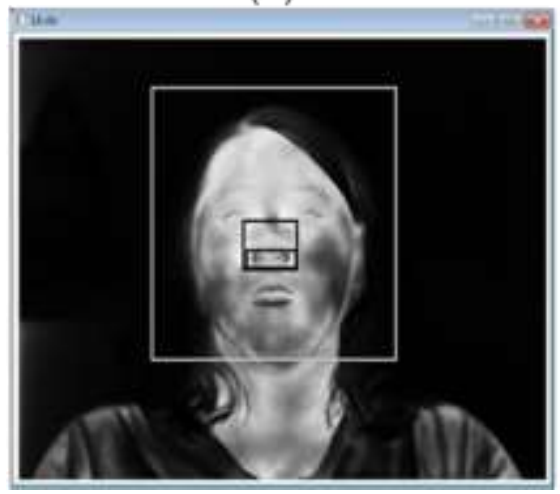

(B)

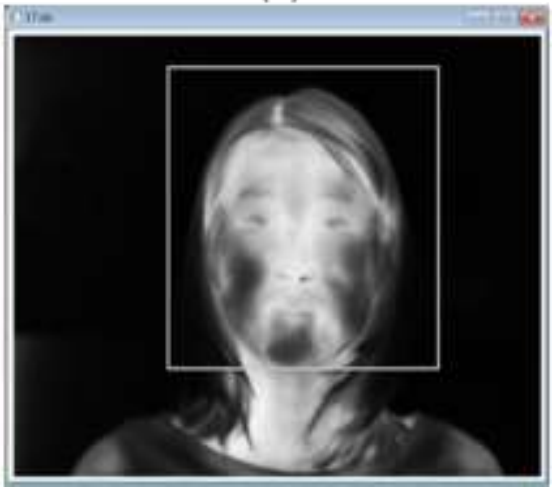

Figure 2. Examples of Correct Detection and Miss for Nose and Nose Hole: (A) Correct Detection (B) Miss 


\subsection{Nose Hole Detection, Nose Hole Tracking, and Breathing Monitoring}

The nose and nose holes were detected in the validity sample to verify the accuracy of the search procedure for new thermal images. The first thermal image of each subject was used to calculate $\mu$ for grayscale conversion, and subsequent thermal images to detect the nose and nose holes. After accurate detection of the nose and nose holes, nose holes were tracked for one minute. While tracking the nose holes, the average temperature of nose hole areas was calculated for each thermal image. Finally, the relationship between the average temperature change of nose holes and breathing rates was determined.

The nose and nose holes were accurately detected in thermal images of all 20 subjects. As shown in Table 2, the features were identified in the second thermal image for 12 out of the 20 subjects. The most number of thermal images required was thirteen. After detecting nose holes in the thermal images, tracking was accurately performed for all 20 subjects.

Table 2. Nose Hole Detection and Breathing Rates for Validity Sample

\begin{tabular}{|c|c|c|c|}
\hline \multirow{2}{*}{ Subjects } & \multirow{2}{*}{ Nose hole detection } & \multicolumn{2}{|c|}{ Breathing rate (breaths per minute) } \\
\hline & & Nose hole temperature & Breathing sensor \\
\hline 1 & $2^{\text {nd }}$ & 11 & 11 \\
\hline 2 & $2^{\text {nd }}$ & 16 & 16 \\
\hline 3 & $2^{\text {nd }}$ & 19 & 20 \\
\hline 4 & $3^{\text {nd }}$ & 21 & 21 \\
\hline 5 & $2^{\text {nd }}$ & - & 18 \\
\hline 6 & $2^{\text {nd }}$ & 13 & 13 \\
\hline 7 & $3^{\text {nd }}$ & 16 & 16 \\
\hline 8 & $11^{\text {nd }}$ & 14 & 13 \\
\hline 9 & $2^{\text {nd }}$ & 12 & 12 \\
\hline 10 & $2^{\text {nd }}$ & 12 & 12 \\
\hline 11 & $4^{\text {nd }}$ & 22 & 22 \\
\hline 12 & $2^{\text {nd }}$ & 13 & 13 \\
\hline 13 & $5^{\text {nd }}$ & 15 & 15 \\
\hline 14 & $2^{\text {nd }}$ & 18 & 18 \\
\hline 15 & $4^{\text {nd }}$ & 13 & 13 \\
\hline 16 & $2^{\text {nd }}$ & 21 & 21 \\
\hline 17 & $2^{\text {nd }}$ & 16 & 16 \\
\hline 18 & $2^{\text {nd }}$ & 15 & 15 \\
\hline 19 & $13^{\text {nd }}$ & 14 & 14 \\
\hline 20 & $8^{\text {nd }}$ & - & 19 \\
\hline
\end{tabular}

Periodic temperature changes of the tracked nose holes were used to calculate breathing rates. To verify the validity of breathing monitoring, breathing rates obtained from respiratory sensors attached to the chest were compared with calculated results. As presented in Table 2, there was a match for 16 out of the 20 subjects, and 2 subjects had different breathing rates for one measurement only. For the remaining 2 subjects, it was difficult to calculate breathing rates based on 
temperature because there was only a small change in temperature near the nose holes, as indicated in Figure 3.

(A)

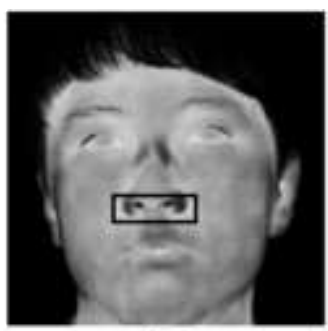

(B)

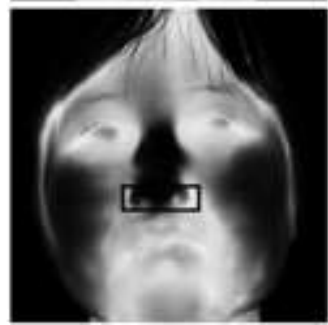



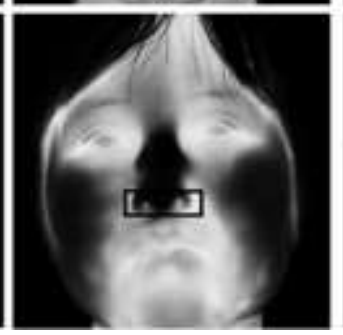

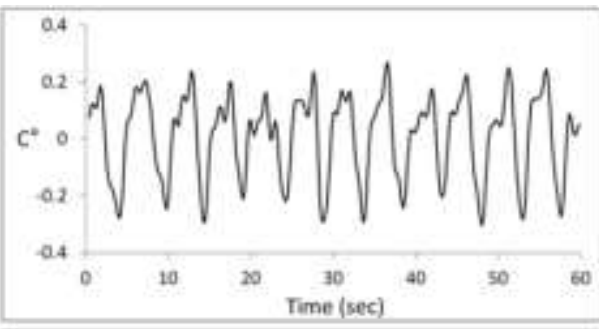

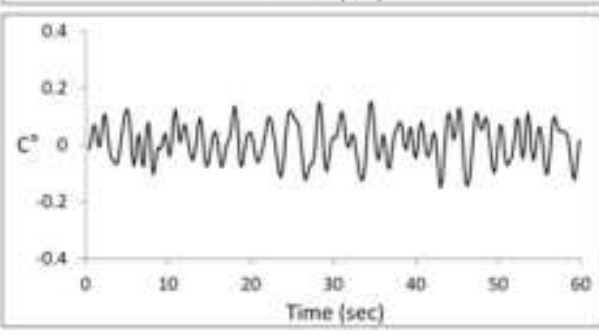

Figure 3. Temperatures of Nose Hole in Inhalation and Exhalation: (A) An Example of Large Temperature Difference (B) An Example of Small Temperature Difference

\section{Conclustion}

This study proposed a method to detect the nose and nose holes from thermal images using the Viola-Jones method, which is based on Haar-like features and the Adaboost algorithm. By applying the proposed method to the validity sample, the nose and nose holes could be accurately detected in all samples. The detected nose hole areas were then used in tracking of the nose holes in thermal images, and breathing rates were calculated by the average temperature of tracked areas. The possibility of monitoring breathing rates with changes in average temperature of nose holes was demonstrated.

The validity of the proposed method was only verified for the front area of the face. This method can be applied to situations involving temperature-based lie detectors as such devices are used on persons facing the front. However, it will be less useful in sleep respiration studies where subjects may be on their sides. This method can be improved to detect the nose and nose holes in faces not facing the front.

\section{Acknowledgments}

This research was supported by the Converging Research Center Program through the Ministry of Science, ICT and Future Planning, Korea (2013K000332).

\section{References}

[1] M. M. Khan, R. D. Ward, and M. Ingleby, "Capturing physiology of emotion along facial muscles: A method of distinguishing feigned from involuntary expressions", In: International Conference on Computer Analysis of Images and Patterns. Springer, Berlin, Heidelberg, (2009), pp. 1196-1203.

[2] Z. Liu, and S. Wang, "Emotion recognition using hidden Markov models from facial temperature sequence", Affective computing and intelligent interaction (2011), pp. 240-247.

[3] L. Trujillo, G. Olague, R. Hammoud, and B. Hernandez, "Automatic feature localization in thermal images for facial expression recognition", In: Computer Vision and Pattern Recognition-Workshops, 2005. CVPR Workshops. IEEE Computer Society Conference on. IEEE, (2005), pp. 14-14.

[4] Y. Yoshitomi, "Facial expression recognition for speaker using thermal image processing and speech recognition system", In: Proceedings of the 10th WSEAS international conference on Applied computer science. World Scientific and Engineering Academy and Society (WSEAS), (2010), pp. 182-186. 
[5] I. Pavlidis, N. L. Eberhardt, and J. A. Levine, "Human behaviour: Seeing through the face of deception", Nature, vol. 415, (2002), 35-35.

[6] D. A. Pollina, A. B. Dollins, S. M. Senter, T. E. Brown, I. Pavlidis, J. A. Levine, and A. H. Ryan, "Facial skin surface temperature changes during a concealed information test", Annals of Biomedical Engineering, vol. 34, no. 7, (2006), pp. 1182-1189.

[7] L. Warmelink, L. Vrij, S. Mann, S. Leal, D. Forrester, and R. P. Fisher, "Thermal imaging as a lie detection tool at airports", Law and Human Behavior, vol. 35, no. 1, (2011), pp. 40-48.

[8] I. Pavlidis, J. Levine, and P. Baukol, "Thermal imaging for anxiety detection", In: Computer vision beyond the visible spectrum: Methods and applications, 2000. Proceedings. IEEE workshop on. IEEE, (2000), pp. 104-109.

[9] R. Murthy, I. Pavlidis, P. Tsiamyrtzis, "Touchless monitoring of breathing function", In: Engineering in Medicine and Biology Society, 2004. IEMBS'04. 26th Annual International Conference of the IEEE. IEEE, (2004), pp. 1196-1199.

[10] J. Fei, and I. Pavlidis, "Thermistor at a distance: unobtrusive measurement of breathing", IEEE Transactions on Biomedical Engineering, vol. 57, no. 4, (2010), pp. 988-998.

[11] F. Q. Al-Khalidi, R. Saatchi, D. Burke, and H. Elphick, H., "Tracking human face features in thermal images for respiration monitoring", In: Computer Systems and Applications (AICCSA), 2010 IEEE/ACS International Conference on. IEEE, (2010), pp. 1-6.

[12] D. Hanawa, T. Morimoto, S. Terada, T. Sakai, and S. Shimazaki, "Nose detection in far infrared image for non-contact measurement of breathing", In: Biomedical and Health Informatics (BHI), 2012 IEEEEMBS International Conference on. IEEE, (2012), pp. 878-881.

[13] P. Viola, and M. Jones, "Robust real-time face detection", International journal of computer vision, vol. 57, no. 2, (2004), pp. 137-154. 
International Journal of Advanced Science and Technology

Vol.109 (2017) 